\title{
Stigma-Based Rejection and the Detection of Signs of Acceptance
}

Social Psychological and

Personality Science

$1-8$

(C) The Author(s) 2015

Reprints and permission:

sagepub.com/journalsPermissions.nav DOI: $10.1177 / 1948550615598376$ spps.sagepub.com

(SAGE

\author{
Laura Smart Richman', Julie Martin', and Jennifer Guadagno²
}

\begin{abstract}
After people experience social rejection, one tactic to restore a sense of belonging is to selectively attend to and readily perceive cues that connote acceptance. The multimotive model of responses to rejection suggests that contextual features of the rejection are important determinants of how people are motivated to respond. According to this model, when rejection is construed as pervasive and chronic, people will be less likely to adopt strategies that promote belonging. Across two studies, we found that chronic rejection -in the context of stigmatization-predicted a slower response time to smiling faces and less recognition of affiliation-related words as compared to a nonstigmatized control group. These results suggest that, unlike more transitory forms of rejection, stigmatization leads to slower detection of signs of acceptance. These responses may hinder belonging repair and thus have important negative implications for health and well-being.
\end{abstract}

\section{Keywords}

stigma, rejection, affiliation

Being rejected is an aversive, unpleasant event. When subjected to various forms of rejection in laboratory manipulations, people experience hurt feelings, depressed affect, anger, emotional numbness, failure of self-regulation, and other negative effects (see Richman \& Leary, 2009, for review). These negative reactions arise because rejection represents a threat to our fundamental need to be valued and accepted by others. This motive is thought to have its origins in evolutionary needs to function within social groups and depend on other people for survival. When this need is threatened by rejection, people are typically motivated to regain acceptance, either from the rejector(s) or from other people. This belongingness restoration can be accomplished by repairing the relationship or seeking new ones. Even when actual sources of acceptance are not available (as may be the case in experimental settings when the immediate support of a friend is not accessible), people may engage in perceptual and cognitive processes that can temporarily restore a sense of belonging (e.g., Gardner, Pickett, Jefferis, \& Knowles, 2005).

The process of searching for indications of one's level of acceptance and belonging can lead people to be particularly attuned to facial cues that communicate acceptance. Nonverbal communication is an integral aspect of human interaction, and although many different features and parts of the body convey emotions, the face is the most informative channel by which people communicate happiness, anger, or sadness (Ekman \& Friesen, 1967). In addition to emotion, the head, face, and eyes supply information about motivational states and the direction of attention, which can be used to infer intentions and the nature of possible future interactions (Lundqvist, Esteves, \& Ohman, 1999; Parkinson, 2005). Accordingly, people often look to the face to gauge the success or failures of social interactions and to determine whether they are being welcomed or rejected.

Following an experience of fleeting rejection, people are particularly motivated to focus on smiling faces. In a series of studies, DeWall, Maner, and Rouby (2009) found that people who experienced the threat of social exclusion were more attuned to smiling faces than those who were not excluded. According to DeWall et al., this attunement may be the result of early-stage cognitive processes that are aimed at achieving compensatory social acceptance in the wake of rejection. Smiles may serve as cues to acceptance, and focusing on them may enable people to feel a renewed sense of belonging. Other studies also find that after experiencing rejection, excluded participants are better able to distinguish genuine (Duchenne) versus fake (non-Duchenne) smiles (Bernstein, Young, Brown, Sacco, \& Claypool, 2008) and prefer working with people who display real from fake smiles (Bernstein, Sacco, Brown,

\footnotetext{
' Department of Psychology and Neuroscience, Duke University, Durham, NC, USA

${ }^{2}$ Facebook, Menlo Park, CA, USA
}

\section{Corresponding Author:}

Laura Smart Richman, Department of Psychology and Neuroscience, Duke University, Soc/Psyc building, Box 90086, Durham, NC 27708, USA.

Email: Irichman@duke.edu 
Young, \& Claypool, 2010). Thus, people may be attuned to smiles because they signal which individuals might be likely sources of acceptance.

Rejected participants not only attune to smiling faces but are also more likely to perceive positivity in ambiguous-expression faces. Maner, DeWall, Baumeister, and Schaller (2007) found that people perceive more kindness and friendliness in neutralexpression faces following rejection. This bias toward perceiving that others are especially welcoming and friendly facilitates efforts to affiliate with them, which increases the likelihood of actual belonging repair. Previous research on self-fulfilling prophecies confirms that entering an interaction with the belief that the other person likes you increases the likelihood of that interaction going well (Merton, 1957; Shelton \& Richeson, 2005).

Orienting toward signs of acceptance is not the response that should predominate in all rejection experiences though. Theorizing on the multiple motives that are activated in response to rejection (Richman \& Leary, 2009) suggests that the way in which rejection is construed influences how people are motivated to respond to the rejection. Even though the motive of gaining acceptance and restoring a sense of belonging following rejection is strong, certain characteristics of the experience may dampen this response or cause other motives to predominate. According to Richman and Leary's multimotive model (2009), when rejection is construed as being pervasive and chronic, people may be less motivated to pursue social reconnection. Expecting rejection from others - based on either prior rejection from that person or because of a disposition to expect less pleasant interactions - prevents people from orienting toward and readily perceiving acceptance cues following rejection (Maner, DeWall, Baumeister, \& Schaller, 2007). Similarly, when people are stigmatized, their identities are linked to undesirable characteristics that may lead to rejection across numerous social contexts (Link \& Phelan, 2001). These rejection experiences then lead to expectations of future rejection in interpersonal interactions that can have specific influences on behavior. If past experience has shown that social acceptance is unlikely, then searching for such cues to affiliation may serve little purpose.

Attention to acceptance cues is an important rejection response to examine because it represents a way of responding to one's social environment that, over time, increases the likelihood of acceptance. People with stigmatized identities may be missing out on opportunities for affiliation and belonging repair if they are setting the criterion for detecting acceptance cues too high. Failure to fulfill the acceptance need over time has critical negative implications for health and well-being by increasing risk for cardiovascular disease, compromised immune function, depression, and other health problems (Pascoe \& Richman, 2009).

Previous research on responses to stigmatization has focused almost exclusively on sensitivity to threat and not on sensitivity to acceptance cues. These studies have found that people high in stigma consciousness and stigma-based rejection sensitivity are more vigilant to threat (Mendoza-Denton,
Purdie, Downey, \& Davis, 2002; Pinel, 1999), and the socialidentity threat literature (e.g., Steele, Spencer, \& Aronson, 2002) suggests that experiencing rejection would make people hypervigilant to further signs of rejection in addition to signs of acceptance. However, studies have consistently found that fleeting rejections do not increase attunement to negatively valenced faces (DeWall, Maner, \& Rouby, 2009) or perceptions of hostility in neutral-expression faces (Maner et al., 2007). Furthermore, there is evidence to suggest that identitybased rejection may not increase vigilance to threat either. For instance, people high and low in fear of negative evaluation did not differ in their ratings of perceived hostility in pictures of upcoming interaction partners, even though they did differ in their ratings of perceived sociability (Maner et al., 2007), suggesting that expectations of rejection may not increase vigilance to threat. Additional research suggests that people with social anxiety avoid and orient away from, rather than toward, signs of social threat (Horley, Williams, Gonzalez, \& Gordon, 2003) precisely because they are so fearful of it. The question still remains, however, as to whether people experiencing stigma-based rejections would display this same response. For these reasons, we examined whether identity-based rejections increase attention toward signs of rejection as well as acceptance.

The primary purpose of the current studies is to extend previous research by examining how stigmatization influences affiliative responses. In Study 1, participants recalled either an identity relevant rejection or wrote about a neutral topic before completing a reaction time task that assessed how quickly they detected smiles on morphing faces. In Study 2, we included a different outcome measure to expand the generalizability of the effect: a word completion task consisting of affiliation-related words. Given the stable attributions that can be made following an identity-based rejection, we hypothesized that when stigmatized status was made salient, participants would be slower to and less likely to detect signs of acceptance as compared to a control group who did not experience any rejection (Study 1) and as compared to a group that experienced nonstigma-based rejection (Study 2). As a secondary interest, we also examined participants' reaction times to signs of hostility in morphing faces (Study 1) and recognition of avoidance-related words (Study 2) to test whether stigmabased rejection increases vigilance to threat. Based on the conflicting findings in the vigilance to threat literature, we entertained the hypothesis that the stigma-salient group would be more vigilant to threat as well as the hypothesis that there would be no difference between groups.

\section{Study I}

Study 1 examined whether participants whose stigmatized status was made salient would be slower to detect the onset of smiling faces as compared to a nonstigmatized group. Participants who were members of at least one stigmatized social group wrote about either an experience where they were treated negatively based on some aspect of their identity or a neutral 

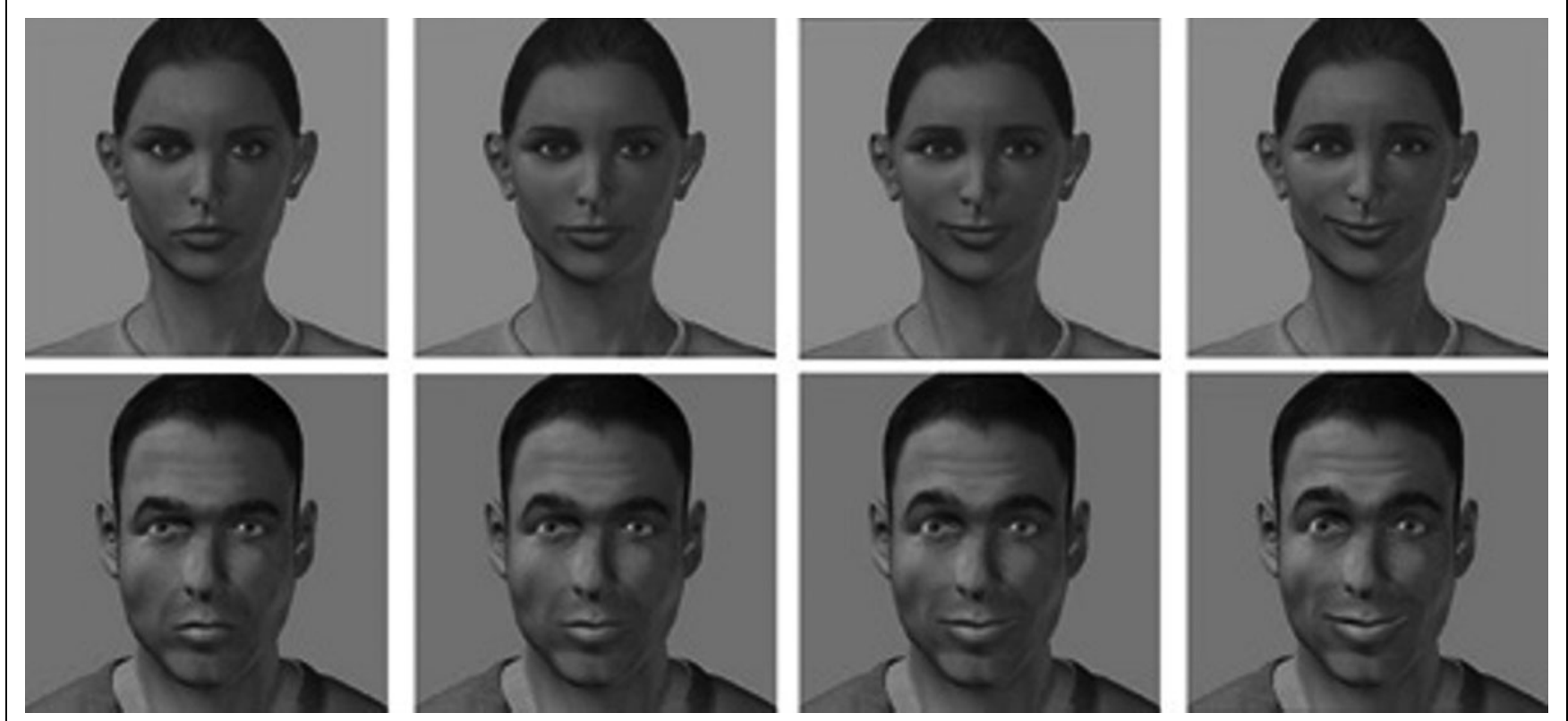

Figure I. Four frames of female (top) and male (bottom) faces morphing from a neutral expression to a smile. The figure is a gray-scale depiction of the faces, although the original images were full color.

subject. We note that the participants in the study possessed stigmatized identities that differ on attributes such as concealability and severity of stigma. Based on previous studies that examined these same identities (Oyserman, Uskel, Yoder, Nesse, \& Williams, 2007), we expected the affiliative motives to operate similarly across stigma groups. Affect was also measured to assess whether response times on the face morph task could be accounted for by differences in emotional responses to the stigma salience manipulation.

\section{Method}

\section{Participants}

Participants indicated in a group testing session whether they were members of a stigmatized group based on the selection criteria used by Oyserman, Uskel, Yoder, Nesse, and Williams (2007). These included minority race or ethnicity, nonheterosexual orientation, low socioeconomic status, physically disabled, or past or current mental health problems. Sixty undergraduate students ( 38 female) met criteria of being part of a stigmatized social group and participated in this study as part of their psychology course requirement.

\section{Materials and Procedure}

Face morph stimuli. The face morph videos used in this study were created with Poser 7, an animation software program used to create three-dimensional characters that allows for the modification of the target's pose, facial structure, skin color, hairstyle, and facial expression. Male and female faces were created and closely matched for facial expression and attractiveness. These faces were white and young, around mid-20s to early 30s (see Figure 1). Consistent with previous studies using face morph methodology (Hugenberg \& Bodenhausen, 2003; Ishii, Miyamoto, Niedenthal, \& Mayama, 2011; Kang \& Chasteen, 2009), movies were created that displayed the target's facial expression slowly changing over a 15 -s time period from a neutral expression to either an expression of contempt or a smile. In total, four different faces were used, each for three trials: male neutral to contempt, female neutral to contempt, male neutral to smile, and female neutral to smile.

Measure of affect. The Positive and Negative Affect Schedule (PANAS) was used to assess mood. This version of PANAS consists of 29 affect-related words, 14 positive and 15 negative. Participants were asked to indicate the degree to which they felt each emotion at the present moment on a 5-point Likert-type scale ranging from 1 (not at all) to 5 (extremely). The different valenced words were combined to form composites of positive and negative emotion $(\alpha=.87$ to .89$)$.

\section{Procedure}

Each participant was randomly assigned to either a stigmasalient condition $(n=32)$ or a neutral control condition $(n=28)$. Participants in the stigma-salient experimental condition were given 10 minutes to write about an experience in the past year in which they felt they were treated differently or discriminated against based on some aspect of their identity. Participants in the control condition wrote a description of the room they were sitting in. After 10 minutes of writing, all participants completed the face morph task using DirectRT software (Version 2008.1.13; Empirisoft Corporation; New York, NY). Participants viewed three trials each of four faces 
previously described. All faces were presented in a random order. Participants were asked to press the space bar on the keyboard the moment they saw an emotion starting to form on the face. The faces all started as neutral and changed to either hostile or smiling expressions. They participated in one practice trial and then began the actual task. After the face morph task, participants completed the PANAS and were then debriefed and dismissed.

\section{Results and Discussion}

Reaction times on the face morph tasks served as the dependent variables. Two participants were removed from analysis for failing to follow instructions. Specifically, a participant in the neutral condition wrote about the meaninglessness of life instead of the room he was sitting in and a participant in the stigma-salient condition wrote that she had never been discriminated against. Three participants were removed for reaction time scores falling 2.5 or more standard deviations $(S D \mathrm{~s})$ above or below the mean for each task. Thus, 55 participants were included in the analysis.

Consistent with previous studies (DeWall et al., 2009; Maner et al., 2007), independent $t$-tests were performed to examine the difference between the experimental and the control condition in detecting the onset of emotion. The difference between conditions to detect a smile (neutral to smile) was significant, with participants in the experimental condition detecting smiling faces slower than participants in the control condition $\left(M_{\text {experimental }}=5,654 \mathrm{~ms}, S D=1,742 \mathrm{~ms}\right.$; $\left.M_{\text {control }}=4,712 \mathrm{~ms}, S D=1,443 \mathrm{~ms}\right), t(52)=-2.13$, $p=.038, d=.59$. The conditions did not differ in their mean time to detect contempt (neutral to contempt), $t(53)=-.39$, $n s\left(M_{\text {experimental }}=5,804 \mathrm{~ms}, S D=1,128 \mathrm{~ms} ; M_{\text {control }}=\right.$ $5,664 \mathrm{~ms}, S D=1,555 \mathrm{~ms})$.

There were no significant differences between conditions on either self-reported positive affect, $t(38)^{1}=-.55, n s$, or negative affect, $t(38)=-.28, n s$, indicating that the differences in response time were not attributable to fluctuations in mood. Although we expected decreased mood to be a consequence of writing about a past rejection experience, ruling out a mood effect strengthens our argument that thinking about rejection as a stable and chronic outcome is the primary driver of slower acceptance cue recognition.

In order to determine whether essay content predicted reaction time, we coded the essay responses on whether participants wrote about a specific instance of rejection based on their identity or a more general experience of being treated differently or discriminated against based on their identity. Two independent coders who were blind to study hypotheses coded the essays on whether they contained a specific instance of rejection, and any interrater discrepancies were resolved through discussion $(K=$ $.56, p<.01)$. Seventeen of the 30 participants in the experimental condition were found to have written about a specific rejecting experience. We reran the analysis excluding participants who wrote about more general experiences, and the same effects emerged as before. Participants in the experimental condition were significantly slower to recognize smiling faces than control participants, $t(39)=-2.51, p=.016, d=.77\left(M_{\text {experimental }}=\right.$ $\left.6,064 \mathrm{~ms}, S D=2,015 \mathrm{~ms} ; M_{\text {control }}=4,712 \mathrm{~ms}, S D=1,443 \mathrm{~ms}\right)$, but not significantly different than the control group on recognition of hostility, $t(40)=-.62$, ns $\left(M_{\text {experimental }}=5,941 \mathrm{~ms}\right.$, $\left.S D=1,183 \mathrm{~ms} ; M_{\text {control }}=5,664 \mathrm{~ms}, S D=1,555 \mathrm{~ms}\right)$.

A smile is a particularly powerful "cue of affiliation intent" (Bernstein et al., 2010). When members of stigmatized groups recalled and wrote about a time when they were discriminated against based on their identity, they were slower to respond to smiling faces, as compared to writing about a neutral experience. The findings from Study 1 suggest that the motive to seek affiliation cues that typically gets activated in response to rejection does not occur when the rejection is related to a stable aspect of the self. When reminded of these experiences, people are instead relatively slow to respond to the cues of acceptance. This study provides preliminary evidence that rejection based on stigma may activate different motives than other experiences of exclusion that have been examined in past research (e.g., DeWall et al., 2009).

\section{Study 2}

Study 2 was a conceptual replication of Study 1 but instead of measuring attention to acceptance cues, we measured the extent to which affiliation and avoidance concepts were salient following an experience of stigmatization. To the extent that a person is searching for acceptance or avoidance cues, these cues should be more salient and he or she should be more likely to see affiliation words in word fragments (Edwards \& Pearce, 1994; Knowles \& Gardner, 2008; Schimel, Greenberg, \& Martens, 2003). We included a fleeting rejection condition (nonstigma based) in addition to a neutral and a stigma-based rejection condition. This allows us to replicate the finding from Study 1 and also examine our hypothesis that stigma-based rejection and nonstigma-based rejection differentially affect recognition of acceptance cues.

\section{Participants and Procedure}

One hundred and thirty-six U.S. residents (66 males, 69 females, and 1 no response) participated for pay. The mean age was $39(S D=13)$. All participants self-identified as gay $(n=49)$, lesbian $(n=21)$, bisexual $(n=64)$, or pansexual $(n=4)$ and were fluent in English. Participants were recruited by Qualtrics, a national survey company that recruits nationwide samples of adults. Participants were given the URL for the online study once they were screened for age and sexual orientation. They were compensated by receiving points that they could then redeem with Qualtrics for gift cards.

Consent was obtained online before the survey was started. Participants were then randomly assigned to a neutral, stigma, or nonstigma-based rejection condition. Participants in the neutral condition were asked to write about the room they were sitting in at the time they completed the survey. Participants in the stigma condition were asked to write about an experience when they "felt rejected due to some aspect of their sexual 
orientation, as a lesbian, gay, bisexual, or transgendered individual." Participants in the nonstigma (fleeting rejection) condition were asked to write about an experience when they "felt rejected due to some occurrence that had nothing to do with their identity." For this condition, they were provided with the example of not being included in group dinner plans because a friend forgot to tell them about it. All participants were given $5 \mathrm{~min}$ to reflect and write in detail about the room or their personal experience with one of these situations.

Participants then completed a word completion task (see Appendix). Words were presented one at a time and for each word, one or more letters was missing. Participants were asked to select a letter that made a word (e.g., if the stem is " $L$ they could choose "a" for LAKE or "i" for LIKE). Each stem had multiple letters that could complete the word. Some of the words were related to affiliation (e.g., share, relate, and attach), some were related to avoidance (e.g., flee, end, and leave), and some were neutral filler words (e.g., print, taste, and beam). Participants completed six words in each category for a total of 18 items.

Participants in all three conditions then answered a few questions about their experience and feelings regarding the writing task. Finally, participants completed the PANAS (same measure as Study $1 ; \alpha_{\text {neg. affect }}=.95, \alpha_{\text {pos. affect }}=.95$ ).

\section{Results and Discussion}

We first reviewed participants' essays to ensure that all participants followed the essay-writing directions. We then conducted one-way analyses of covariance to examine the effect of the experimental manipulation on completion of the word fragments to form affiliation- and avoidance-related words. Number of completed words on the neutral trials was included as a covariate to control for participants' general ability to complete the word fragments. A main effect of condition was found for the completion of affiliation-related words, $F(2,133)=$ $3.06, p=.05, \eta_{p}^{2}=.04$ ). Planned pairwise comparisons (Fisher's least significant difference) revealed that participants in the stable rejection condition were significantly less likely to complete the word fragments with affiliation-related words $(M=2.15, S D=.91)$ than participants in the fleeting rejection condition $(M=2.69, S D=1.15 ; p=.017)$, and marginally less likely to spell affiliation-related words than participants in the control condition $(M=2.51, S D=1.00 ; p=.083)$. Furthermore, the difference between the control group and the fleeting rejection group was in the predicted direction, though it was not significant (see Figure 2). There were no differences between the groups on likelihood of completing word fragments with avoidance-related words, $M_{\text {stable }}=2.37, S D=1.16 ; M_{\text {fleeting }}=$ $2.29, S D=1.25 ; M_{\text {control }}=2.31, S D=1.17 ; F(2,133)=.04, n s$.

To ensure that the intensity of the rejection essays did not vary as a function of condition, independent raters who were blind to study hypotheses rated the intensity $(1=$ not at all, $2=$ moderately, and $3=$ very) of the stable and fleeting rejection essays $(K=.62, p<.01)$, and any disagreements were resolved through discussion. A $t$-test revealed no significant

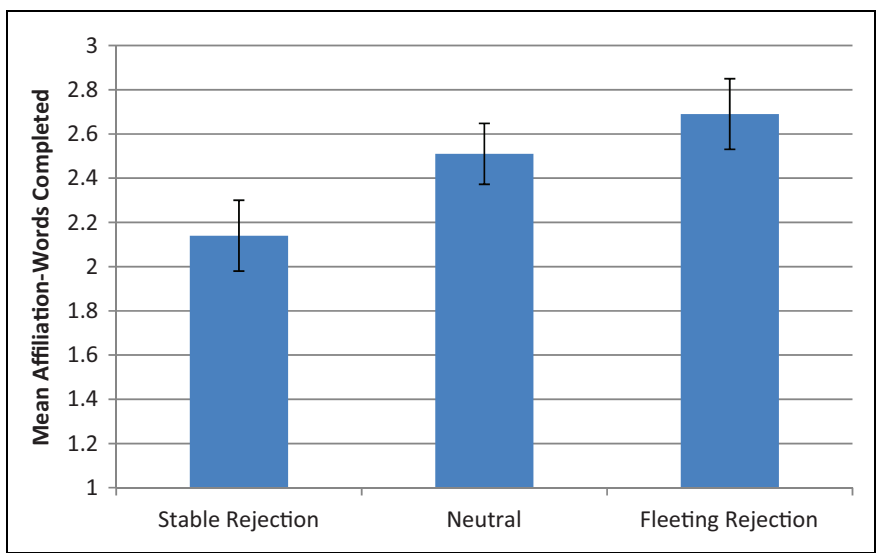

Figure 2. Study 2: Effect of condition on mean number of word fragments completed with affiliation-related words.

difference between the fleeting and the stable rejection conditions as a function on intensity, $t(79)=-.41, n s$.

There was a significant main effect of condition on negative affect, $F(2,133)=4.99, p=.008 ; M_{\text {control }}=1.40, S D=.63$; $\left.M_{\text {stable }}=1.92, S D=.96 ; M_{\text {fleeting }}=1.68, S D=.87\right)$, but not positive affect, $F(2,133)=2.20, n s$, so we reran the analyses for affiliation words with affect included as a covariate. We found the same results as before, $F(2,130)=2.98, p=.05$, suggesting that the effect of condition on affiliation-word completion is not attributable to a mood effect.

These findings provide further evidence suggesting that people orient differently to affiliation cues depending on the features of the rejection experience. Using a different outcome measure (orienting to affiliation-related words), similar results were found to the previous study regarding the difference between a stigma-salient group and control group. When participants were stigmatized, they were less likely to think of and recognize affiliation-related words as compared to participants who experienced nonidentity-based rejection and as compared to participants in a neutral, no rejection condition.

\section{General Discussion}

Prior research has established that people search for signs of acceptance following rejection (DeWall et al., 2009; Maner et al., 2007). The primary mechanism thought to underlie these effects is that people are motivated to restore a sense of value and acceptance following a threat to their belonging needs, and orienting toward smiling faces that communicate acceptance is one way to fulfill this need. However, theorizing on multiple motives that are activated in response to rejection (Richman \& Leary, 2009) suggests that the context in which rejection is experienced, and the way in which it is construed, determines whether affiliation is the predominate motive that is triggered. In this study, we provide evidence that when people are stigmatized - a type of rejection that is chronic and pervasive $^{2}$ - they are relatively slow to react to signs of acceptance. We also find that, following an experience of being stigmatized, affiliation-related words are less salient in people's 
minds compared to a fleeting rejection group and a norejection control group.

One way to consider these findings is that recouping social losses may be regarded as more of a possibility when the rejection is not based on immutable aspects of one's identity. The multimotive model predicts that people are motivated to act in prosocial ways when the possibility for relationship repair exists. In Study 2, when the rejection was not based on a stable aspect of their identity, people responded relatively rapidly to affiliation words. In contrast, when rejection was based on one's stigmatized identity, people may have set higher standards for what constitutes indications of acceptance.

Much of the work on stigmatization and other chronic forms of discrimination has focused on how prior experiences of mistreatment may condition people to expect future mistreatment. These expectations, coupled with a desire to minimize the possibility of missing signs of potential rejection, are thought to cause stigmatized groups to be overly attuned to indications of rejection. Although intuitive, this hypothesis has yet to be directly supported. Previous research has focused on individual differences in identity-based rejection sensitivity and stigma consciousness, finding that these dispositions increase the detection of social threat (Inzlicht, Kaiser, \& Major, 2008; London, Rattan, Downey, Romero-Canyas, \& Tyson, 2012). However, experimental research has not found that stigmabased rejection itself increases vigilance, and our findings suggest that stigma-based rejection decreases attention to acceptance cues without increasing focus on rejection cues. This finding is consistent with Maner et al.'s (2007) work showing that people high in fear of negative evaluation rated pictures of upcoming interaction partners as less sociable following rejection but did not differ from low fear of negative evaluation participants in their ratings of perceived hostility of upcoming partners following rejection. Thus, the current research suggests that, rather than increasing vigilance to threat, stigmabased rejections prevent the ready recognition of acceptance cues by raising the threshold for detecting signs of affiliation.

Approach and avoidance motivational systems have long been hypothesized to be relatively independent (e.g., Gray, 1987). Thus, one can be high or low in approach motives as well as high or low in avoidance motives (Gable, 2006), and these motivations can vary across situations (Elliot, 2008). An important consideration when determining if a person will pursue approach or avoidance goals is how feasible the person thinks each of the goals is (Brunstein, 1993; Elliot, 2008). Although our data suggest that the stigmatized are less likely than the nonstigmatized to view affiliation-related approach goals as feasible, they may not view avoiding further rejection as particularly feasible either. This consideration may help to explain why participants did not increase in avoidance motivation following stigma-based rejection. Future work should further examine the contexts in which stigma-based rejection may activate these different motives, but the current studies support prior research findings that people can be high or low in both orientations in any given context (Elliot, 2008; Gable, 2006).
The group differences in negative affect found in Study 2, but not Study 1, suggest that the essay-writing manipulation had a stronger effect on mood in Study 2. This may be due to the fact that participants in Study 2 were explicitly asked to describe how the rejection made them feel, which may have made their emotional state more salient (participants in Study 1 were not asked to engage in this reflection). Furthermore, because Study 2 consisted only of lesbian, gay, and bisexual adults who knew they were being recruited based on their sexual identity, their stable rejection essays were mostly stories of coming out to close family and friends who then rejected them. Participants in Study 1, on the other hand, possessed various different stigmatized identities and primarily wrote about rejections within the past year from strangers. Although rejections from strangers also affect mood in the moment, reflecting on those experiences later may have less of an effect on mood than recalling rejections from close family and friends. This explanation is consistent with Schmitt, Branscombe, Postmes, and Garcia's (2014) meta-analysis showing that possessing a stigmatized identity that is less socially acceptable (such as one that even friends and family would not accept) is associated with greater decreases in general affect and well-being. Even though a mood effect was not found in Study 1, the manipulation was successful in making participants' stigmatized identities salient, which was the main objective of the manipulation and the hypothesized driver of the decreased attention to acceptance cues.

Rejected individuals have a strong need to reaffiliate and restore a sense of belonging. Our findings suggest that when one is stigmatized, the need to reaffiliate may be present, but the behaviors that may encourage the fulfillment of this need are not there. This response may play a critical role in better understanding how members of groups that are stigmatized may be vulnerable to negative long-term effects on health and well-bring. If orienting toward signs of acceptance is indeed an early-stage cognitive process that enables rejected people to reestablish social connections, then our findings suggest that stigmatized people may be missing such opportunities. While previous theorizing has focused on sensitivity to threat as a precursor to health and well-being difficulties for stigmatized populations, the current research suggests that inattentiveness to acceptance cues may be the more prevalent and therefore more problematic response to stigma-based rejection.

\section{Appendix}

\section{Study 2: Word Completion Task Word Fragments}

Affiliation Words:
S H A E
RE_A T E
O I N
A T T A C
EN_A GE
D E_E N D
Avoidance Words 


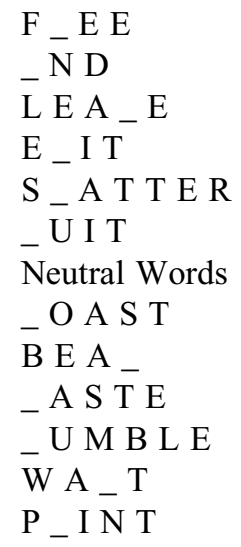

\section{Declaration of Conflicting Interests}

The author(s) declared no potential conflicts of interest with respect to the research, authorship, and/or publication of this article.

\section{Funding}

The author(s) disclosed receipt of the following financial support for the research, authorship, and/or publication of this article: This research was supported in part by grants from the National Science Foundation and the National Institutes of Mental Health and Guadagno NSF \#DGF1106401.

\section{Notes}

1. The first 15 participants were not given the Positive and Negative Affect Schedule, so the $d f$ is lower than the overall $d f$ for the other tests.

2. We suggest that individuals who are part of a stigmatized group perceive discrimination based on their stigmatized group membership to be chronic and pervasive. In a separate sample, we asked 90 students about possible stigmatizing group memberships (race, religion, and being overweight) and asked them to rate the degree to which they felt they were discriminated based on their stigmatized status, how often they perceived discrimination, and in how many different domains of life they felt discriminated against because of their stigma. We found that feeling as though one is discriminated against based on race, religion, or weight is associated with experiencing these discriminating experiences as pervasive $\left(r_{\text {race }}=.83, p<.001 ; r_{\text {religion }}=.88, p<.001 ; r_{\text {weight }}=.71 ; p<\right.$. $001)$ and chronic $\left(r_{\text {race }}=.74, p<.001 ; r_{\text {religion }}=.76, p<.001\right.$; $\left.r_{\text {weight }}=.74, p<.001\right)$.

\section{References}

Bernstein, M. J., Sacco, D. F., Brown, C. M., Young, S. G., \& Claypool, H. M. (2010). A preference for genuine smiles following social exclusion. Journal of Experimental Social Psychology, 46, 196-199.

Bernstein, M. J., Young, S. G., Brown, C. M., Sacco, D. F., \& Claypool, H. M. (2008). Adaptive responses to social exclusion: Social rejection improves detection of real and fake smiles. Psychological Science, 19, 981-983.

Brunstein, J. C. (1993). Personal goals and subjective well-being: A longitudinal study. Journal of Personality and Social Psychology, 65, 1061-1070.
DeWall, C. N., Maner, J. K., \& Rouby, D. A. (2009). Social exclusion and early-stage interpersonal perception: Selective attention to signs of acceptance. Journal of Personality and Social Psychology, 96, 729-741.

Edwards, L. C., \& Pearce, S. A. (1994). Word completion in chronic pain: Evidence for schematic representation of pain? Journal of Abnormal Psychology, 103, 379-382.

Ekman, P., \& Friesen, W. V. (1967). Head and body cues in the judgment of emotion: A reformulation. Perceptual and Motor Skills, 24, 711-724.

Elliot, A. J. (2008). Handbook of approach and avoidance motivation. New York, NY: Psychology Press.

Gable, S. L. (2006). Approach and avoidance social motives and goals. Journal of Personality, 74, 175-222.

Gardner, W. L., Pickett, C. L., Jefferis, V., \& Knowles, M. (2005). On the outside looking in: Loneliness and social monitoring. Personality and Social Psychology Bulletin, 31, 1549-1560.

Gray, J. A. (1987). The psychology of fear and stress. New York, NY: Cambridge University Press.

Horley, K., Williams, L. M., Gonzalvez, C., \& Gordon, E. (2003). Social phobics do not see eye to eye. A visual scanpath study of emotional expression processing. Journal of Anxiety Disorders, $17,33-44$.

Hugenberg, K., \& Bodenhausen, G. V. (2003). Facing prejudice: Implicit prejudice and the perception of facial threat. Psychological Science, 14, 640-643. doi:10.1046/j.0956-7976.2003.psci_ 1478.x

Inzlicht, M., Kaiser, C. R., \& Major, B. (2008). The face of chauvinism: How prejudice expectations shape perceptions of facial affect. Journal of Experimental Social Psychology, 44, 758-766.

Ishii, K., Miyamoto, Y., Mayama, K., \& Niedenthal, P. M. (2011). When your smile fades away: Cultural differences in sensitivity to the disappearance of smiles. Social Psychological and Personality Science, 2, 516-522. doi:10.1177/1948550611399153

Kang, S. K., \& Chasteen, A. L. (2009). Beyond the double-jeopardy hypothesis: Assessing emotion on the faces of multiplycategorizable targets of prejudice. Journal of Experimental Social Psychology, 45, 1281-1285. doi:10.1016/j.jesp.2009.07.002

Knowles, M. L., \& Gardner, W. L. (2008). Benefits of membership: the activation and amplification of group identities in response to social rejection. Personality \& Social Psychology Bulletin, 34, 1200-1213. doi:10.1177/0146167208320062

Link, B., \& Phelan, J. (2001). Conceptualizing stigma. Annual Review of Sociology, 27(3), 363-385.

London, B., Rattan, A., Downey, G., Romero-Canyas, R., \& Tyson, D. (2012). Gender-based rejection sensitivity and academic selfsilencing in women. Interpersonal Relations and Group Processes, Journal of Personality and Social Psychology, 102, 961-972.

Lundqvist, D., Esteves, F., \& Ohman, A. (1999). The face of wrath: Critical features conveying facial threat. Cognition and Emotion, 13, 691-711.

Maner, J. K., DeWall, C. N., Baumeister, R. F., \& Schaller, M. (2007). Does social exclusion motivate interpersonal reconnection? Resolving the "porcupine problem." Journal of Personality and Social Psychology, 92, 42-55. 
Mendoza-Denton, R., Purdie, V., Downey, G., \& Davis, A. (2002). Sensitivity to status-based rejection: Implications for AfricanAmerican students' college experience. Journal of Personality and Social Psychology, 83, 896-918.

Merton, R. (1957). Social theory and social structure. Glencoe, IL: Free Press.

Oyserman, D., Uskel, A. K., Yoder, N., Nesse, R. M., \& Williams, D. R. (2007). Unfair treatment and self-regulatory focus. Journal of Experimental Social Psychology, 43, 505-512.

Parkinson, B. (2005). Do facial movements express emotions or communicate motives? Personality and Social Psychology Review, 9, 278-311.

Pascoe, E., \& Richman, L. (2009). Perceived discrimination and health: A meta-analytic review. Psychological Bulletin, 135, 531-554.

Pinel, E. C. (1999). Stigma consciousness: The psychological legacy of social stereotypes. Journal of Personality and Social Psychology, 76, 114-128.

Richman, L. S., \& Leary, M. (2009). Reactions to discrimination, stigmatization, ostracism, and other forms of interpersonal rejection: A multi motive model. Psychological Review, 116, 365-383.

Schimel, J., Greenberg, J., \& Martens, A. (2003). Evidence that projection of a feared trait can serve a defensive function. Personality and Social Psychology Bulletin, 29, 969-979.

Schmitt, M. T., Branscombe, N. R., Postmes, T., \& Garcia, A. (2014). The consequences of perceived discrimination for psychological well-being: A meta-analytic review. Psychological Bulletin, 140, 921-948.
Shelton, J. N., \& Richeson, J. A. (2005). Intergroup contact and pluralistic ignorance. Journal of Personality and Social Psychology, 88, 91-107.

Steele, C. M., Spencer, S. J., \& Aronson, J. (2002). Contending with group image: The psychology of stereotype and social identity threat. Advances in Experimental Social Psychology, $34,379-440$

\section{Author Biographies}

Laura Smart Richman is an assistant professor of psychology and neuroscience at Duke University. She is a social and health psychologist, and her research examines how people respond to discrimination, social hierarchies, and lack of power. She focuses on cognitive, behavioral, and physiological responses that have implications of health behaviors and outcomes.

Julie Martin is a fourth-year graduate student in social psychology at Duke University. Her research examines how people respond to social rejection emotionally, cognitively, and behaviorally, and how contextual and personality factors affect those responses to rejection.

Jennifer Guadagno is a researcher at Facebook. As a social psychologist, she examines how scientifically supported theory and processes can be used to encourage more mindful, compassionate, and supportive interactions online. Her work focuses on applying evidencebased practices to support people going through difficult life experiences (suicidal ideations, bullying, and mental and physical illness) that show up online. 